\title{
A Simple Analysis of Anand, GTN and J-C Constitutive Models for Meso - damage Mechanics
}

\author{
Cui Tian-xiao ${ }^{1, a)}$
}

School of Ordnance Engineering University, Shijiazhuang 050003, China

a)245616722@qq.com

Keywords: Analysis, Anand, GTN, J-C constitutive model, Meso-damage

\begin{abstract}
Based on the damage mechanics of materials, the definition and content of meso damage mechanics are introduced, and then the meso damage constitutive model is derived. On the basis of meso damage constitutive model, The characteristics and applicability of Anand meso damage model, Gurson-Tvergaard-Needleman (GTN) meso damage model and Johnson-Cook (J-C) meso damage constitutive model were analyzed respectively.
\end{abstract}

\section{O INTRODUCTION}

Since the beginning of the Industrial Revolution, the status of materials in scientific research is becoming the most important research topic in the 21st century, all walks of life need to use a variety of materials to meet the material production and social development. With the progress of science, all kinds of materials to meet the various properties are improving, but there is no perfect material, with the load and the role of the environment, any material will produce damage and corrosion. Therefore, in the analysis of material performance life, the need to consider the material damage changes in order to accurately determine the use of.

\section{DEFINITION AND CONNOTATION OF MESO - DAMAGE MECHANICS}

Damage mechanics is a new discipline developed in recent decades. In the role of the load and the environment, due to micro-structural defects (such as micro-cracks, micro-holes) caused by material and structural degradation process, known as damage. Damage mechanics is to study the material properties of damaged media, as well as damage in the deformation process of evolution until the destruction of the mechanical processes of discipline ${ }^{[1]}$

Damage mechanics has two main branches: one is continuous medium mechanics, which uses continuous media thermodynamics and continuum mechanics of the phenomenological method to study the damage of the mechanical process. It focuses on the impact of damage on the macro-mechanical properties of materials and the evolution of material and structural damage. The second is the meso-damage mechanics. Based on the deformation and evolution of damage elements, such as micro-cracks, micro-holes, shear bands and various kinds of primitive elements, according to the deformation and evolution of damaged elements, through a mechanical averaging method, The relationship between material deformation and damage process and meso-damage parameters is obtained $^{[2,3]}$.

The meso-damage mechanics is based on the meso-structure of materials, and different meso-damage mechanisms are distinguished, through the study of the physical and mechanical processes of meso-structure changes, the damage of the material is known, and the macroscopic properties of the materials were deduced from the micro-analysis results by volume averaging method. The meso-damage mechanics uses the methods of continuum mechanics and material science to describe the meso-structures such as microvoids, microcracks and grain boundaries. There are many kinds of meso-damage mechanisms,Micro-cracks, micro-slip bands, crazing, grain boundary slip, etc ${ }^{[4]}$.

Microvoid damage of ductile metallic materials. Ductile metal damage damage process can be divided into the following three stages:

Micropores are nucleated. The nucleation of micropores is mainly due to the inhomogeneity of 
microstructure, most of the micropores are nucleated near the two-phase particles, or self-cracking generated in the two-phase particles, or in the interface between the two-phase particles and the substrate.

Microporous growth. With the constant loading, microvoids around the plastic deformation of the material is growing, micro-holes also will be expanded and grown up.

The confluence of microvoids. When the plastic deformation near the micro-hole reaches a certain level, the plastic instability occurs between the micro-holes, resulting in the local shear band between the micro-holes. The secondary pores in the local shear band converge to form macroscopic cracks

\section{DAMAGE MODEL BASED ON ANAND CONSTITUTIVE EQUAYION}

In the unified viscoplastic constitutive model, the most widely used Anand model ${ }^{[5-7]}$. It has the characteristics of simple form and few model parameters. It has been widely used in the life prediction of electronic solder joint. The Anand model uses a single internal variable $\mathrm{S}$ related to dislocation density, solid solution strengthening and grain size effect to describe the macroscopic impedance of the internal state of the material to plastic flow. It can reflect the deformation behavior of visco-plastic material related to strain rate and temperature, as well as the historical effect of strain rate, strain hardening and dynamic recovery. However, the Anand model does not consider the coupling of deformation and damage, that is, the stress-strain relationship does not change due to damage evolution.

Uniform viscoplastic Anand constitutive model has two basic characteristics ${ }^{[5-7]}$ : (1) There is no explicit yield surface in the stress space, so no loading / unloading criterion is required during the deformation process. Plastic deformation occurs under all non-zero stress conditions. (2) A single internal variable is used to describe the macroscopic impedance of the internal state of the material to the plastic flow. Internal variables (or deformation resistance) are marked with $\mathrm{S}$ and have a stress dimension.

The flow equation of viscoplastic Anand model uses the hyperbolic creep law to predict the rate dependence and temperature dependency of the material. The expression is as follows

$$
\begin{gathered}
\sigma=C:\left[\varepsilon-\varepsilon_{P}-I \alpha\left(T-T_{0}\right)\right] \\
\dot{\varepsilon}_{P}=\frac{3}{2} A e^{\left(-\frac{Q}{R T}\right)}\left[\sinh \left(\xi \frac{\sqrt{\frac{3}{2} s: s}}{S}\right)\right]^{\frac{1}{m}} \cdot \frac{s}{\sqrt{\frac{3}{2} s: s}} \\
\dot{S}=\left\{h_{0}\left|1-\frac{S}{S^{*}}\right|^{a} \operatorname{sign}\left(1-\frac{S}{S^{*}}\right)\right\} \cdot \sqrt{\frac{2}{3} \dot{\varepsilon_{P}}: \dot{\varepsilon_{P}}} \\
S^{*}=\stackrel{\Delta}{S}\left[\frac{\sqrt{\frac{2 \dot{\varepsilon_{P}}: \dot{\varepsilon_{P}}}{A}} e^{\frac{Q}{R T}}}{A}\right]^{n}
\end{gathered}
$$

Where: $\sigma$ is the Cauchy stress, $s$ is the partial stress, $C$ is the elastic tensor, $\varepsilon$ is the total strain, $\alpha$ is the thermal expansion coefficient, $\dot{\varepsilon}_{p}$ is the inelastic strain rate, $A$ is the constant, $Q$ is the activation energy, $m$ is the strain sensitivity index, $\xi$ is the stress multiplier, $R$ is the gas constant, $T$ is the absolute temperature, $T_{0}$ is the reference temperature, $h_{0}$ is the strain hardening-softening constant, $a$ is the strain sensitivity coefficient associated with hardening and softening, $S^{*}$ is the variable saturation value, $\stackrel{\Delta}{S}$ is the coefficient, and $n$ is an exponent.

\section{GTN MESI-DAMAGE MODEL}

Gurson proposed a meso-damage constitutive model for ductile porous materials, which can be used to describe macroscopic material softening and cracking due to the growth of pores, nucleation 
and polymerization. Compared with the concept of incompressible plastic volume in traditional plasticity model, it considers the effect of macroscopic hydrostatic stress on yield surface. Since then, Tvergaard and Needleman have modified the Gurson model, the revised model is the GTN model, expressed as

$$
\Phi=\left(\frac{\sigma_{e q}}{\sigma_{m}}\right)+2 q_{1} f^{*} \cosh \left(-\frac{3}{2} \times \frac{q_{2} \sigma_{h}}{\sigma_{m}}\right)-\left(1+q_{3} f^{* 2}\right)=0
$$

In the formula: $\sigma_{e q}=\sqrt{\frac{3}{2} S_{i j} S_{i j}}$, is the macroscopic Mises equivalent stress; $S_{i j}=\sigma_{i j}-\frac{1}{3} \sigma_{k k} \delta_{i j}$, represents the deviatoric stress component of the macroscopic stress; $\sigma_{i j}$ is the macroscopic stress component; $\sigma_{k k}$ is the macroscopic principal stress component; $\delta_{i j}$ for the Kronecker mark; $\sigma_{h}=-\frac{1}{3} \sigma_{k k}$, for the macro hydrostatic stress; $\sigma_{m}$ is the equivalent stress of the matrix material; $q_{1}, \quad q_{2}$ and $q_{3}$,three additional fitting parameters were introduced for Tvergaard, when $q_{1}=q_{2}=q_{3}=1$, the GTN model degrades to the original Gurson model; $f^{*}$ as the damage variable, Is a function of the pore volume fraction $f$.

GTN meso-damage model is based on micromechanics, and it is believed that any material in the process of the existence of defects in the process (such as inclusion, holes, etc., most of the GTN model using holes for damage modeling, that hole is the initial damage), These defects are affected by the loading level and stress state (stress triaxiality) during the loading process. The voids begin to evolve (nucleation, expansion and contraction of the damage, resulting in the formation of fine cracks) and eventually the formation of macroscopic macroscopic cracks. The GTN model was developed by extending the initial Gurson meso-damage model, which models the meso-scale scale with an equivalent averaging technique, Mesoscale loading is used to study the change of meso-physical quantities, mesoscale as an intermediary to study the evolution of mesoscale physical quantities on macroscopic scale material properties evolution, so mesoscale - macroscopic scale mechanics model is completed. The model has a lot of literature in the academic community also carried out a related study.

\section{J-C DAMAGE CONSTITUTIVE MODEL}

Johnson-Cook constitutive model is one of the most widely used constitutive models in numerical simulation of impact dynamics. The model expresses the flow stress as the product of strain hardening $f_{1}\left(\varepsilon_{p}\right)$, strain rate strengthening $f_{2}(\dot{\varepsilon})$, and heat softening $f_{3}(T)$, specific form

$$
\sigma\left(\varepsilon_{P}, \dot{\varepsilon}, T\right)=\left[A+B \varepsilon_{P}^{n}\right]\left[1+C \ln \dot{\varepsilon} / \dot{\varepsilon}_{0}\right]\left[1-T^{* m}\right]
$$

In the formula: $A$-reference strain rate and initial yield stress at reference temperature $T_{r} ; \quad B$ and $n$-material strain hardening modulus and hardening index; $C$-material strain rate strengthening parameter; $m$-material thermal softening index; $T^{*}$-homologous temperature 。

Johnson-Cook Damage Constitutive Model Based on the experiment, the constitutive equation and its parameters are obtained by fitting the experimental parameters. The advantage of this model is that each physical quantity of the model has definite physical meaning, and the comparison of the model parameters is easily obtained through experiments, is now widely used in academic and industrial a phenomenological damage model, a large number of literature to describe it and related experimental verification studies, and is suitable for metal damage research, is a relatively mature metal damage modeling tool.

\section{CONCLUSION}

The damage constitutive laws of the three materials are Anand meso-damage model, GTN meso-damage model and Johnson-Cook damage constitutive model. These three models are 
respectively the damage constitutive of different mechanisms, which have been applied successfully in academia and industry. The latter two models are phenomenological damage models, but their modeling ideas and modeling starting point are different. By applying different constitutive models, a more complete and complete performance life analysis of the material can be carried out.

\section{REFETENCES}

[1] Yu Tian-qing, Qian Ji-cheng. Damage Theory and Its Application [M]. Beijing: National Defense Industry Press, 1993.

[2] Lauro F., Bennani B., Croix P. , et al., Identification of the Damage Parameters for Anisotropic Materials by Inverse Technique: Application to an Aluminium[J]. Journal of Materials Processing Technology, 2001, 118(1-3):472-477.

[3] Johnson G. R., Cook W. H.. Fracture Characteristics of Three Metals Subjected to Various Strains, Strain Rates, Temperatures and Pressures[J]. Engineering Fracture Mechanics, 1985, 21:31-48.

[4] Borvik T., Hopperstad O. S., Berstad T.. On the Influence of Stress Triaxiality and Strain Rate On the Behaviour of a Structural Steel. Part II. Numerical Study[J]. European Journal of Mechanics A/Solids, 2003, 22:15-32.

[5] Brown S B, Kim K H, Anand L. An internal variable constitutive model for hot working metals [ J] .International Journal of Plastici ty,1989, 5: 95- 130.

[6] Gomez J, Basaran C. Damage mechanics constitutive model for $\mathrm{Pb} / \mathrm{Sn}$ solder joints incorporating nonlinear kinematic hardening and rate dependent effects using a return mapping integration algor ithm[ J] . Mechanics of Mater ials, 2006, 38: 585- 598.

[7] Wang D J, PantoR L. Experimental study of void formation in eutectic and lead free solder bumps of flip chip assemblies [ J] . Journal of Electronic Packaging, 2006, 128: 202- 207. 